\title{
Systematic Review \\ The Prevalence of Sarcopenia in Chinese Older Adults: Meta-Analysis and Meta-Regression
}

\author{
Zi Chen ${ }^{(D)}$, Wei-Ying Li ${ }^{D}$, Mandy Ho and Pui-Hing Chau *(D) \\ School of Nursing, The University of Hong Kong, Hong Kong, China; u1chenzi@connect.hku.hk (Z.C.); \\ u3003886@connect.hku.hk (W.-Y.L.); mandyho1@hku.hk (M.H.) \\ * Correspondence: phchau@graduate.hku.hk; Tel.: +852-39-176-626
}

Citation: Chen, Z.; Li, W.-Y.; Ho, M.; Chau, P.-H. The Prevalence of Sarcopenia in Chinese Older Adults: Meta-Analysis and Meta-Regression. Nutrients 2021, 13, 1441. https:// doi.org/10.3390/nu13051441

Academic Editors: Cristiano Capurso and Maria Luz Fernandez

Received: 9 February 2021

Accepted: 21 April 2021

Published: 24 April 2021

Publisher's Note: MDPI stays neutral with regard to jurisdictional claims in published maps and institutional affiliations.

Copyright: (C) 2021 by the authors Licensee MDPI, Basel, Switzerland. This article is an open access article distributed under the terms and conditions of the Creative Commons Attribution (CC BY) license (https:// creativecommons.org/licenses/by/ $4.0 /)$.

\begin{abstract}
Sarcopenia, with risk factors such as poor nutrition and physical inactivity, is becoming prevalent among the older population. The aims of this study were (i) to systematically review the existing data on sarcopenia prevalence in the older Chinese population, (ii) to generate pooled estimates of the sex-specific prevalence among different populations, and (iii) to identify the factors associated with the heterogeneity in the estimates across studies. A search was conducted in seven databases for studies that reported the prevalence of sarcopenia in Chinese older adults, aged 60 years and over, published through April 2020. We then performed a meta-analysis to estimate the pooled prevalence, and investigated the factors associated with the variation in the prevalence across the studies using meta-regression. A total of 58 studies were included in this review. Compared with community-dwelling Chinese older adults (men: 12.9\%, 95\% CI: 10.7-15.1\%; women: $11.2 \%, 95 \%$ CI: $8.9-13.4 \%$ ), the pooled prevalence of sarcopenia in older adults from hospitals (men: $29.7 \%$, 95\% CI:18.4-41.1\%; women: $23.0 \%, 95 \%$ CI:17.1-28.8\%) and nursing homes (men: $26.3 \%, 95 \%$ CI: 19.1 to $33.4 \%$; women: $33.7 \%, 95 \%$ CI: 27.2 to $40.1 \%$ ) was higher. The multivariable metaregression quantified the difference of the prevalence estimates in different populations, muscle mass assessments, and areas. This study yielded pooled estimates of sarcopenia prevalence in Chinese older adults not only from communities, but also from clinical settings and nursing homes. This study added knowledge to the current epidemiology literature about sarcopenia in older Chinese populations, and could provide background information for future preventive strategies, such as nutrition and physical activity interventions, tailored to the growing older population.
\end{abstract}

Keywords: sarcopenia; prevalence; nutrition; physical activity; meta-analysis; meta-regression

\section{Introduction}

The speed of population ageing is accelerating globally. According to statistics from the World Health Organization (WHO), by 2050, the proportion of individuals over the age of 60 is expected almost to double (22\%) compared with 12\% in 2015 [1]. China is already witnessing this demographic trend; thus, the country's National Bureau of Statistics has predicted that the number of adults aged 65 years and above will increase from 166.58 million (11.9\% of the total population) in 2018 to 366 million in 2050 [2].

Advancing age is marked by a series of physiological changes in body composition, including the decrease in skeletal muscle mass and increase in fat mass [3,4]. Sarcopenia is the age-related decline in skeletal muscle mass and function characterized by the loss of muscle strength and physical performance [5]. Accordingly, understanding more about its etiology and risk factors is of great interest. The onset and progression of sarcopenia can be attributed to numerous factors including physical inactivity and poor nutrition [6]. Therefore, most non-pharmacological interventions about sarcopenia mainly target these two modifiable factors. An umbrella review concluded that exercise training, especially resistance training, had a significant effect on the improvement of muscle mass, muscle strength, and physical performance [7]. Bloom et al. systematically reviewed observational 
evidence regarding the relationship of diet quality and sarcopenia, and concluded that a higher quality diet was associated with better physical performance among older adults [8]. Furthermore, a narrative review conducted by Tessier et al. examined the observational and interventional evidence regarding the association between some specific nutrients and sarcopenic components [9]. They found that some nutrients such as proteins, leucine, vitamin $\mathrm{D}$, and $\mathrm{n}-3$ polyunsaturated fatty acids (n-3 PUFAs) might have a protective impact on muscle health among older adults [9]. Previous research has associated sarcopenia with such adverse health outcomes as fractures, falls, functional decline, hospitalization, and even increased mortality $[10,11]$. Hence, early screening and identifying sarcopenia among older populations should be at the forefront of timely diet and/or exercise interventions for sarcopenia prevention and treatment.

Sarcopenia is diagnosed based on low muscle mass, low muscle strength, and diminished physical performance. However, no standard and unique diagnosis criteria for sarcopenia have yet been established. At present, several international groups, such as the European Working Group on Sarcopenia in Older People (EWGSOP), Asia Working Group for Sarcopenia (AWGS), International Working Group on Sarcopenia (IWGS), Foundation for the National Institutes of Health (FNIH) Sarcopenia Project, have provided their own diagnostic criteria for sarcopenia [12-14]. Estimates of sarcopenia prevalence are, in turn, dependent on the diagnostic criteria used to define it. For example, a longitudinal multicenter cohort research found the prevalence of sarcopenia among community-dwelling older adults ranged from $3.3 \%$ to $17.5 \%$ depending on the diagnostic criteria used (specifically, AWGS: 9.1\%, EWGSOP: 17.5\%, IWGS: 16.1\%, and FNIH: 3.3\%) [15]. Assessment of muscle mass is an essential part of sarcopenia diagnosis. Dual-energy X-ray absorptiometry (DXA) and bioelectrical impedance analysis (BIA) are both recommended to assess muscle mass in research and practice [12]. Therefore, estimates of the prevalence may also depend on different assessment approaches. For example, Beaudart et al. [16] compared the prevalence of sarcopenia (EWGSOP criterion) using different muscle mass assessments (DXA and BIA) among older adults over 65 years, and found the prevalence was lower when using the BIA technique (BIA vs. DXA: $12.8 \%$ vs. $21 \%$ ). Likewise, there is evidence that the methods used to measure muscle strength and physical performance may yield inconsistent estimates of the prevalence of sarcopenia [17]. Furthermore, estimates of prevalence also varied across populations and areas [18-23]. Previous evidence revealed that hospitalized older adults and nursing-home residents had higher prevalence of sarcopenia compared with community-dwelling older residents [18]. Community-dwelling Chinese older adults residing outside mainland China (i.e., Hong Kong and Taiwan) showed a lower prevalence rate of sarcopenia than counterparts from the mainland [24].

At present, two systematic reviews have pooled the estimate of sarcopenia prevalence in community-dwelling Chinese older adults (17\% and $11 \%$, respectively) [24,25]. However, few systematic reviews have pooled the prevalence for Chinese older adults in other settings, such as clinical settings and nursing homes. Furthermore, no study has systematically investigated the factors contributing to the heterogeneity in the estimates of sarcopenia prevalence through meta-regression. Therefore, we conducted a meta-analysis and meta-regression to shed light on the prevalence of sarcopenia in Chinese older adults not only from communities, but also from clinical settings and nursing homes, and to explain the heterogeneity in sarcopenia prevalence across studies.

Information about the prevalence of sarcopenia is the first step to develop preventive routines or health services tailored to the growing older population. Meta-analysis and meta-regression of prevalence data are increasingly important for policy making and implementation of preventive measures in situations for which inconsistent prevalence estimates have been reported in the literature. This study could help policy makers and health practitioners make evidence-based decisions targeting the health issues of sarcopenia. 


\section{Methods}

This review was conducted in accordance with the Preferred Reporting Items for Systematic Reviews and Meta Analyses (PRISMA) statement [26].

\subsection{Search Methods}

Two researchers (Z.C. and W.Y.L.) independently searched the following online electronic databases: PubMed, Cochrane Library, Embase (Ovid), CINAHL, Web of Science Core Collection, CNKI, and Wanfang (accessed on 15 April 2020); the latter two being Chinese. The search period was restricted from the earliest records to 15 April, 2020. Key search terms were "sarcopenia", "epidemiology", "prevalence", "aged", "elderly", "older", "China", and "Chinese". Logical operators (i.e., AND, OR) were used to shape the search strategy. Detailed search strategies for PubMed and Wanfang are presented in the Supplementary Materials as examples (S1: Search strategy). Relevant studies were identified through manual searches in the reference list of eligible studies.

Inclusion criteria were as followed: (1) the prevalence of sarcopenia was reported or could be calculated; (2) participants were of Chinese ethnicity; (3) the age was 60 years and over; (4) participants were recruited from the community, clinical settings, or nursing homes; (5) EWGOSP, AWGS, IWGS or FINH definitions of sarcopenia were adopted; (6) muscle mass was measured with DXA or BIA; (7) primary research irrespective of design. Exclusion criteria were (1) studies published as reviews, letters to editors, conference abstracts, expert opinions, case reports; (2) studies published in languages other than English and Chinese.

First, duplicate records were identified and removed. Then, titles and abstracts were screened to remove studies irrelevant to the research questions. Next, full-text articles were retrieved and reviewed for eligibility according to the selection criteria. When multiple papers came from the same dataset, we chose the one with the largest sample size which might be closer to the original cohort in order to avoid those using a particular subset of the whole dataset. The above process was performed independently by the two researchers (Z.C. and W.-Y.L.). Any discrepancies were adjudicated by a third researcher (P.-H.C.).

\subsection{Data Extraction}

The following details were extracted from each of the eligible studies: the year of publication, country or area in which the data were collected, research design, sample size, settings where participants were recruited, diagnostic criteria for sarcopenia, measurement of muscle mass, muscle strength, and physical performance and prevalence of sarcopenia. For cohort or intervention studies, only baseline data were extracted. For studies using more than one diagnostic criterion to define sarcopenia, all estimates of prevalence were extracted.

\subsection{Critical Appraisal}

Two researchers (Z.C. and W.Y.L.) independently assessed the quality of included studies using a validated tool developed by Hoy et al., which was tailored to assess the risk of bias for prevalence studies with different types of design [27]. The tool evaluates the risk of bias through 10 items. The first four items mainly focus on external validity, which involve the representativeness of the target population and the sampling frame, selection of the sample, and non-response bias. The remaining items address the issue of internal validity, which involves the use of proxy respondent or not, acceptable case definition, validated measurement, consistent mode of data collection, the length of the shortest prevalence period, and correct calculation of prevalence. Each item was rated as "low risk" or "high risk". When information in the article was not sufficient for judgement, that item was rated as "high risk". Following previous literature, the study was considered to have a low risk of bias when 9 or 10 items were rated "low risk"; a moderate risk of bias when 6 to 8 items were rated "low risk"; and a high risk of bias when 5 or less items were rated "low risk" [28,29]. 


\subsection{Statistical Analysis}

The prevalence of sarcopenia varied in different genders and populations recruited from different settings [18]. Accordingly, in this study, the pooled prevalence was obtained separately for each gender and for the population from each setting. For studies which recruited participants from mixed settings, only those which reported the prevalence for each setting were included in the meta-analysis. While more than one estimate was extracted from studies which used more than one diagnostic criterion to define sarcopenia, only the one that was more frequently used in other eligible studies was used for calculation of the pooled estimate.

Random-effect models take into account the possibility that the parameters for the population may vary among studies [30]. Therefore, because of the variations in populations, diagnosis criteria, muscle mass assessments, etc., among the eligible studies, we chose a random-effect model to calculate the pooled prevalence. The metaprop command in Stata 15.0 (Stata Corp, College Station, TX, USA) was used to obtain pooled estimates of prevalence [31,32].

We assessed the heterogeneity in the estimates across studies using Cochran's Q test, with a $p$-value $<0.10$ indicating heterogeneity [33]. We quantified the heterogeneity using Isquare, with $\mathrm{I}^{2}$ statistics of $25 \%, 50 \%$, and $75 \%$ set as the cut-offs for low, moderate, and high heterogeneity, respectively [33]. If heterogeneity existed, random-effect meta-regression was conducted to explore the potential source of variability in prevalence estimates across studies. Meta-regression can explore the effects of multiple study characteristics on the variance of pooled estimates simultaneously [30]. For this study, we performed multivariable meta-regression (using the metareg command) to model the adjusted association between multiple explanatory variables and prevalence estimates. Based on previous evidence, we considered the following study characteristics to be potential sources of heterogeneity: populations from different settings, diagnostic criteria for sarcopenia, assessment of muscle mass, muscle strength and physical performance, and the area of study. In order to have a sufficient power, meta-regression was only performed for covariates reported in at least ten studies [33].

Publication bias refers to the phenomenon that studies with significant results are more likely to get published than those with negative results, which can result in systematic differences between published and unpublished studies [34]. In the case of observational studies that report prevalence, however, there are no positive or negative results, and no well-established method is recommended to test for this bias in meta-analysis of prevalence studies. Therefore, in this study, we did not check for publication bias.

\section{Results}

\subsection{Search Outcomes}

We identified 459 records from databases and 6 records from the reference lists. After removing 139 duplicated records, we screened the titles and abstracts of the remaining 326 records, and removed another 168 irrelevant records, leaving 158 studies. Further reviewing the full texts of these studies for eligibility, we excluded another 100 for the reasons specified in Figure 1. We carried out the quantitative synthesis (i.e., meta-analysis and meta-regression) on the remaining 58 records.

The characteristics of the included studies are summarized in Table 1. Fifty-one $(87.9 \%)$ studies were classified as having a moderate risk of bias and seven $(12.1 \%)$ were classified as having a low risk of bias. None of the included studies recruited the nationwide representative sample. Twelve studies adopted random sampling [19,20,35-44]. One study was open to the non-response bias [35], Each study collected data of sarcopenia components directly from subjects and had a consistent mode of data collection. All included studies had acceptable case definition and correct calculation of prevalence. Details of the critical appraisal are presented in Supplementary Materials (Table S2). 


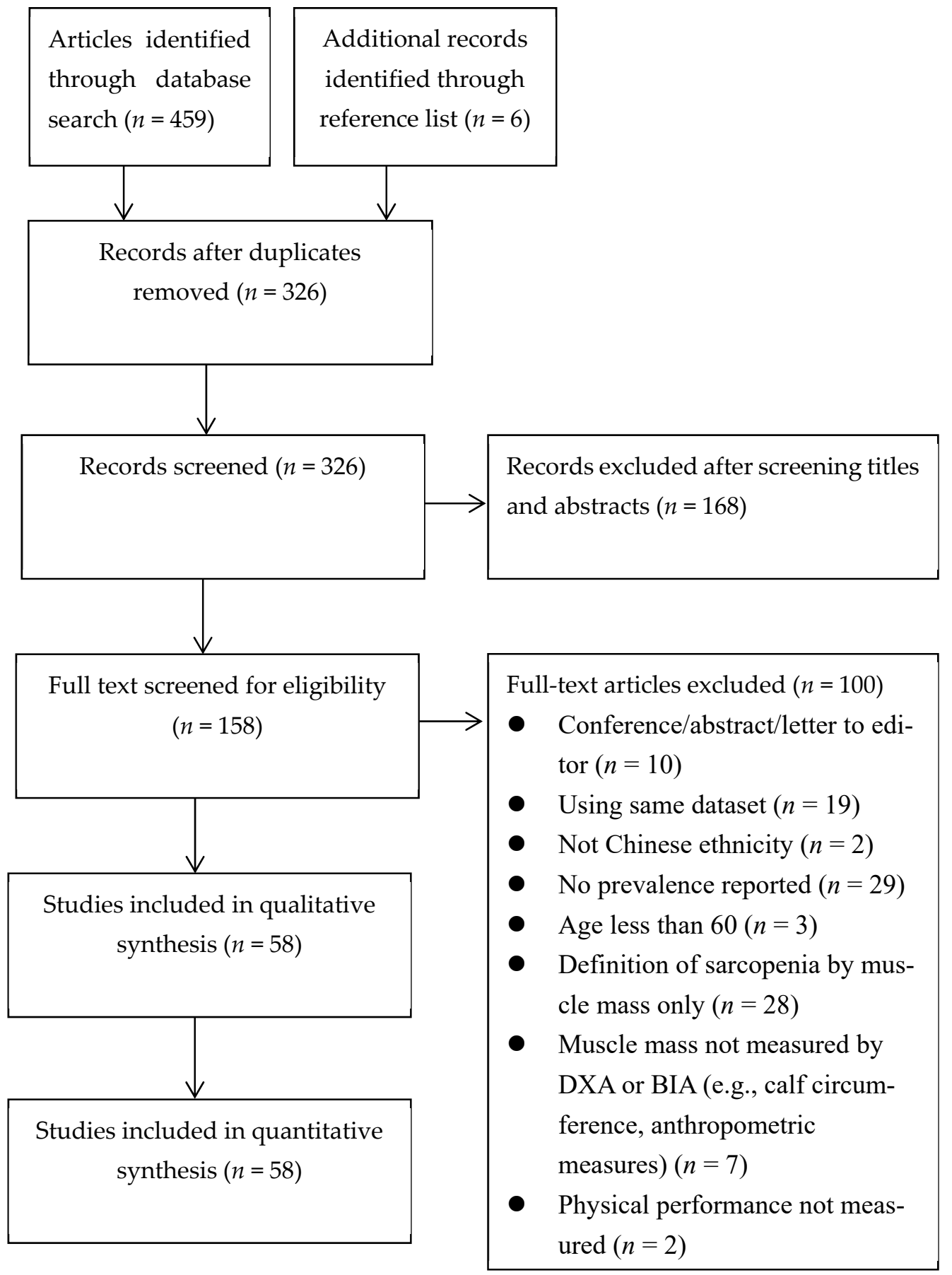

Figure 1. PRISMA flowchart. 
Table 1. Characteristics of included studies $(n=58)$.

\begin{tabular}{|c|c|c|c|c|c|c|c|c|c|c|c|c|c|c|c|}
\hline \multirow{3}{*}{ Study } & \multirow{3}{*}{ Language } & \multirow{3}{*}{ Region } & \multirow{3}{*}{ Design } & \multirow{2}{*}{\multicolumn{3}{|c|}{ Sample Size }} & \multirow{3}{*}{$\begin{array}{l}\text { Diagnostic } \\
\text { Criteria }\end{array}$} & \multicolumn{4}{|c|}{ Assessment } & \multirow{2}{*}{\multicolumn{3}{|c|}{ Prevalence $n(\%)$}} & \multirow{3}{*}{$\begin{array}{l}\text { Risk of } \\
\text { Bias }\end{array}$} \\
\hline & & & & & & & & \multirow{2}{*}{$\begin{array}{c}\text { Muscle } \\
\text { Mass }\end{array}$} & \multirow{2}{*}{$\begin{array}{l}\text { Muscle } \\
\text { Strength }\end{array}$} & \multicolumn{2}{|c|}{ Physical Performance } & & & & \\
\hline & & & & Total & Male & Female & & & & $\begin{array}{c}\text { Distance } \\
\text { (m) }\end{array}$ & Gait Speed & Total & Male & Female & \\
\hline Meng et al., 2014 [45] & English & Mainland & $\begin{array}{c}\text { Cross- } \\
\text { sectional }\end{array}$ & 101 & 101 & - & EWGSOP & DXA & Dynamometer & 6 & Usual & $46(45.7)$ & $46(45.7)$ & - & Moderate \\
\hline Wu et al., 2014 [46] & English & Taiwan & $\begin{array}{c}\text { Cross- } \\
\text { sectional }\end{array}$ & 549 & 285 & 264 & EWGSOP & BIA & Dynamometer & 5 & - & $39(7.1)$ & $11(3.9)$ & $28(10.6)$ & Moderate \\
\hline Zhang et al., 2014 [47] & Chinese & Mainland & $\begin{array}{l}\text { Cross- } \\
\text { sectional }\end{array}$ & 116 & - & - & EWGSOP & DXA & Dynamometer & 6 & Usual & $48(41.4)$ & - & - & Moderate \\
\hline Meng et al., 2015 [35] & English & Taiwan & $\begin{array}{l}\text { Cross- } \\
\text { sectional }\end{array}$ & 771 & 412 & 359 & EWGSOP & DXA & Dynamometer & 5 & Usual & $44(5.7)$ & $35(8.4)$ & $9(2.6)$ & Moderate \\
\hline Wang et al., 2015 [48] & English & Mainland & $\begin{array}{l}\text { Cross- } \\
\text { sectional }\end{array}$ & 316 & 164 & 152 & AWGS & BIA & Dynamometer & 4 & Usual & $94(29.4)$ & $43(26.2)$ & $51(33.6)$ & Moderate \\
\hline Wen et al., 2015 [36] & English & Mainland & $\begin{array}{l}\text { Cross- } \\
\text { sectional }\end{array}$ & 286 & 136 & 150 & $\begin{array}{l}\text { IWGS } \\
\text { EWGSOP } \\
\text { AWGS }\end{array}$ & DXA & Dynamometer & 6 & Usual & $\begin{array}{c}17(5.9) \\
1(0.3) \\
9(3.1)\end{array}$ & $\begin{array}{l}10(7.4) \\
1(0.8) \\
8(5.9)\end{array}$ & $\begin{array}{l}7(4.7) \\
1(0.7)\end{array}$ & Moderate \\
\hline Chan et al., 2016 [19] & English & HK & $\begin{array}{l}\text { Cross- } \\
\text { sectional }\end{array}$ & 3957 & 1979 & 1878 & AWGS & DXA & Dynamometer & 6 & Usual & $290(7.3)$ & $185(9.3)$ & $105(5.6)$ & Low \\
\hline Han et al., 2016 [49] & English & Taiwan & $\begin{array}{l}\text { Cross- } \\
\text { sectional }\end{array}$ & 878 & 402 & 476 & EWGSOP & BIA & Dynamometer & 7 & Usual & $29(3.3)$ & $27(6.7)$ & $2(0.4)$ & Moderate \\
\hline Han et al., 2016 [50] & English & Mainland & $\begin{array}{l}\text { Cross- } \\
\text { sectional }\end{array}$ & 1069 & 467 & 602 & AWGS & BIA & Dynamometer & 4 & Usual & $99(9.3)$ & $30(6.4)$ & $69(11.5)$ & Moderate \\
\hline Wang et al., 2016 [51] & English & Mainland & $\begin{array}{l}\text { Cross- } \\
\text { sectional }\end{array}$ & 944 & 462 & 482 & AWGS & BIA & Dynamometer & 6 & Usual & $98(10.4)$ & $38(8.2)$ & $60(12.5)$ & Moderate \\
\hline Wang et al., 2016 [52] & English & Mainland & $\begin{array}{l}\text { Cross- } \\
\text { sectional }\end{array}$ & 854 & 404 & 450 & AWGS & BIA & Dynamometer & 4 & Usual & $96(11.2)$ & $53(13.1)$ & $43(9.6)$ & Low \\
\hline Xia et al., 2016 [37] & Chinese & Mainland & $\begin{array}{l}\text { Cross- } \\
\text { sectional }\end{array}$ & 683 & 239 & 444 & AWGS & BIA & Dynamometer & 4 & - & $137(20.1)$ & $41(17.2)$ & $96(21.6)$ & Moderate \\
\hline Fang et al., 2017 [53] & Chinese & Mainland & $\begin{array}{l}\text { Cross- } \\
\text { sectional }\end{array}$ & 106 & - & 106 & AWGS & DXA & Dynamometer & 6 & Usual & $13(12.2)$ & - & $13(12.2)$ & Moderate \\
\hline Hai et al., 2017 [54] & English & Mainland & $\begin{array}{c}\text { Cross- } \\
\text { sectional }\end{array}$ & 836 & 415 & 421 & AWGS & BIA & Dynamometer & 6 & Usual & $88(10.5)$ & $47(11.3)$ & $41(9.7)$ & Moderate \\
\hline Hua et al., 2017 [55] & Chinese & Mainland & $\begin{array}{l}\text { Cross- } \\
\text { sectional }\end{array}$ & 300 & 168 & 132 & AWGS & BIA & Dynamometer & 6 & Usual & $54(18.0)$ & $38(22.6)$ & $16(12.1)$ & Moderate \\
\hline Meng et al., 2017 [56] & Chinese & Mainland & $\begin{array}{l}\text { Cross- } \\
\text { sectional }\end{array}$ & 106 & 101 & 5 & AWGS & BIA & Dynamometer & - & - & $29(27.4)$ & - & - & Moderate \\
\hline Chu 2018 [57] & Chinese & Mainland & $\begin{array}{l}\text { Cross- } \\
\text { sectional }\end{array}$ & 191 & 69 & 122 & AWGS & BIA & Dynamometer & 4 & Maximal & $28(14.7)$ & $8(11.6)$ & $20(16.4)$ & Moderate \\
\hline Wang et al., 2018 [23] & English & Mainland & $\begin{array}{l}\text { Cross- } \\
\text { sectional }\end{array}$ & 865 & 427 & 438 & AWGS & BIA & Dynamometer & 6 & Usual & $71(7.1)$ & $28(6.6)$ & $33(7.5)$ & Moderate \\
\hline Yang et al., 2018 [58] & English & Mainland & $\begin{array}{l}\text { Cross- } \\
\text { sectional }\end{array}$ & 384 & 160 & 224 & EWGSOP & BIA & Dynamometer & 4 & Usual & 45 (11.72) & $17(10.6)$ & $28(12.5)$ & Moderate \\
\hline Zhang et al., 2018 [38] & Chinese & Mainland & $\begin{array}{l}\text { Cross- } \\
\text { sectional }\end{array}$ & 1148 & 368 & 780 & AWGS & BIA & Dynamometer & 6 & Usual & $164(14.3)$ & $55(14.9)$ & $109(14.0)$ & Low \\
\hline Chen et al., 2019 [21] & English & Mainland & Prospective & 691 & 304 & 387 & AWGS & BIA & Dynamometer & 4 & - & $55(8.0)$ & - & - & Moderate \\
\hline
\end{tabular}


Table 1. Cont.

\begin{tabular}{|c|c|c|c|c|c|c|c|c|c|c|c|c|c|c|c|}
\hline \multirow{3}{*}{ Study } & \multirow{3}{*}{ Language } & \multirow{3}{*}{ Region } & \multirow{3}{*}{ Design } & \multirow{2}{*}{\multicolumn{3}{|c|}{ Sample Size }} & \multirow{3}{*}{$\begin{array}{c}\text { Diagnostic } \\
\text { Criteria }\end{array}$} & \multicolumn{4}{|c|}{ Assessment } & \multirow{2}{*}{\multicolumn{3}{|c|}{ Prevalence $n(\%)$}} & \multirow{3}{*}{$\begin{array}{c}\text { Risk of } \\
\text { Bias }\end{array}$} \\
\hline & & & & & & & & \multirow{2}{*}{$\begin{array}{c}\text { Muscle } \\
\text { Mass }\end{array}$} & \multirow{2}{*}{$\begin{array}{l}\text { Muscle } \\
\text { Strength }\end{array}$} & \multicolumn{2}{|c|}{ Physical Performance } & & & & \\
\hline & & & & Total & Male & Female & & & & $\begin{array}{c}\text { Distance } \\
(\mathrm{m})\end{array}$ & Gait Speed & Total & Male & Female & \\
\hline Du et al., 2019 [22] & English & Mainland & $\begin{array}{c}\text { Cross- } \\
\text { sectional }\end{array}$ & 631 & 213 & 418 & AWGS & BIA & Dynamometer & 6 & Usual & $77(12.2)$ & 41 (19.2) & $36(8.6)$ & Moderate \\
\hline Liu et al., 2019 [39] & Chinese & Mainland & $\begin{array}{l}\text { Cross- } \\
\text { sectional }\end{array}$ & 1723 & 915 & 808 & AWGS & BIA & Dynamometer & 6 & Usual & $121(7.0)$ & $96(10.5)$ & $25(3.1)$ & Moderate \\
\hline Liu 2019 [59] & Chinese & Mainland & $\begin{array}{l}\text { Cross- } \\
\text { sectional }\end{array}$ & 769 & 416 & 353 & AWGS & BIA & Dynamometer & 6 & Usual & $32(4.16)$ & $12(2.9)$ & $20(5.7)$ & Moderate \\
\hline Wang et al., 2019 [60] & English & Mainland & $\begin{array}{c}\text { Cross- } \\
\text { sectional }\end{array}$ & 945 & 465 & 480 & AWGS & BIA & Dynamometer & 6 & Usual & $276(29.2)$ & $123(26.5)$ & $153(55.4)$ & Moderate \\
\hline Xu et al., 2019 [40] & English & Mainland & $\begin{array}{c}\text { Cross- } \\
\text { sectional }\end{array}$ & 2412 & 1012 & 1400 & AWGS & BIA & Dynamometer & 6 & Usual & $156(6.5)$ & $58(5.7)$ & $98(7.0)$ & Moderate \\
\hline Zhang et al., 2019 [61] & English & Mainland & $\begin{array}{l}\text { Cross- } \\
\text { sectional }\end{array}$ & 1002 & 420 & 582 & AWGS & BIA & Dynamometer & 4 & - & $107(10.7)$ & $37(8.8)$ & $70(12.0)$ & Moderate \\
\hline Liu et al., 2020 [15] & English & Mainland & $\begin{array}{c}\text { Cross- } \\
\text { sectional }\end{array}$ & 1712 & - & - & AWGS & BIA & Dynamometer & 4 & Usual & $556(32.5)$ & - & - & Moderate \\
\hline Rong et al., 2020 [62] & English & Mainland & $\begin{array}{l}\text { Cross- } \\
\text { sectional }\end{array}$ & 450 & 266 & 184 & AWGS & BIA & Dynamometer & 6 & Usual & $89(19.7)$ & $50(18.8)$ & $39(21.2)$ & Moderate \\
\hline Xu et al., 2020 [63] & English & Mainland & $\begin{array}{l}\text { Cross- } \\
\text { sectional }\end{array}$ & 582 & 246 & 336 & AWGS & BIA & Dynamometer & 6 & Usual & $15(526.6)$ & $82(33.3)$ & $73(21.7)$ & Moderate \\
\hline Yang et al., 2020 [64] & English & Mainland & $\begin{array}{l}\text { Cross- } \\
\text { sectional }\end{array}$ & 483 & 184 & 299 & $\begin{array}{c}\text { FNIH } \\
\text { IWGS } \\
\text { AWGS } \\
\text { EWGSOP1 } \\
\text { EWGSOP2 }\end{array}$ & BIA & Dynamometer & 4 & Usual & $\begin{array}{c}16(3.3) \\
78(16.1) \\
44(9.1) \\
76(15.7) \\
22(4.6)\end{array}$ & $\begin{array}{l}11(6.0) \\
45(24.5) \\
20(10.9) \\
41(22.3) \\
12(6.5)\end{array}$ & $\begin{array}{c}5(1.7) \\
33(11.0) \\
24(8.0) \\
35(11.7) \\
10(3.3)\end{array}$ & Moderate \\
\hline \multicolumn{16}{|c|}{ Hospitals $(n=11)$} \\
\hline Wang et al., 2016 [52] $^{\dagger}$ & English & Mainland & $\begin{array}{c}\text { Cross- } \\
\text { sectional }\end{array}$ & 236 & 116 & 120 & AWGS & BIA & Dynamometer & 4 & Usual & $35(14.8)$ & $20(17.2)$ & $15(12.5)$ & Low \\
\hline Cui 2018 [65] & Chinese & Mainland & $\begin{array}{c}\text { Cross- } \\
\text { sectional }\end{array}$ & 132 & 59 & 73 & AWGS & DXA & Dynamometer & 6 & Usual & $38(28.8)$ & $21(35.6)$ & $17(23.3)$ & Moderate \\
\hline Zhai et al., 2018 [66] & English & Mainland & $\begin{array}{c}\text { Cross- } \\
\text { sectional }\end{array}$ & 494 & 216 & 278 & AWGS & DXA & Dynamometer & 6 & - & $158(32.0)$ & $87(40.3)$ & $71(25.5)$ & Moderate \\
\hline Chen et al., 2019 [67] & English & Mainland & $\begin{array}{c}\text { Cross- } \\
\text { sectional }\end{array}$ & 118 & 92 & 26 & AWGS & DXA & Dynamometer & 6 & Usual & $71(60.17)$ & 65 (70.65) & $6(23.08)$ & Moderate \\
\hline Wang 2019 [41] & Chinese & Mainland & $\begin{array}{c}\text { Cross- } \\
\text { sectional }\end{array}$ & 119 & 64 & 55 & AWGS & BIA & Dynamometer & - & - & $26(21.8)$ & $17(26.6)$ & $9(16.3)$ & Moderate \\
\hline Yao 2019 [68] & Chinese & Mainland & $\begin{array}{c}\text { Cross- } \\
\text { sectional }\end{array}$ & 378 & 153 & 225 & AWGS & BIA & Dynamometer & 6 & Usual & 47 (12.4) & $15(9.8)$ & $32(14.2)$ & Moderate \\
\hline Yi et al., 2019 [69] & Chinese & Mainland & $\begin{array}{c}\text { Cross- } \\
\text { sectional }\end{array}$ & 200 & - & - & AWGS & BIA & Dynamometer & 6 & - & $98(49)$ & - & - & Moderate \\
\hline Tan 2019 [70] & Chinese & Mainland & $\begin{array}{c}\text { Cross- } \\
\text { sectional }\end{array}$ & 734 & - & - & AWGS & BIA & Dynamometer & 4 & - & $258(35.1)$ & - & - & Moderate \\
\hline Zhang et al., 2019 [71] & English & Mainland & Prospective & 345 & 208 & 137 & AWGS & BIA & Dynamometer & 6 & - & 78 (22.6) & $32(15.4)$ & 46 (33.6) & Moderate \\
\hline Cui et al., 2020 [72] & English & Mainland & $\begin{array}{l}\text { Cross- } \\
\text { sectional }\end{array}$ & 132 & 59 & 73 & AWGS & DXA & Dynamometer & 6 & Usual & $38(28.8)$ & $21(55.3)$ & $17(44.7)$ & Moderate \\
\hline Wang et al., 2020 [73] & Chinese & Mainland & $\begin{array}{c}\text { Cross- } \\
\text { sectional }\end{array}$ & 236 & 144 & 92 & AWGS & BIA & Dynamometer & 6 & - & $63(26.7)$ & $28(19.4)$ & $35(38.0)$ & Moderate \\
\hline
\end{tabular}


Table 1. Cont.

\begin{tabular}{|c|c|c|c|c|c|c|c|c|c|c|c|c|c|c|c|}
\hline \multirow{3}{*}{ Study } & \multirow{3}{*}{ Language } & \multirow{3}{*}{ Region } & \multirow{3}{*}{ Design } & \multirow{2}{*}{\multicolumn{3}{|c|}{ Sample Size }} & \multirow{3}{*}{$\begin{array}{c}\text { Diagnostic } \\
\text { Criteria }\end{array}$} & \multicolumn{4}{|c|}{ Assessment } & \multirow{2}{*}{\multicolumn{3}{|c|}{ Prevalence $n(\%)$}} & \multirow{3}{*}{$\begin{array}{c}\text { Risk of } \\
\text { Bias }\end{array}$} \\
\hline & & & & & & & & \multirow{2}{*}{$\begin{array}{l}\text { Muscle } \\
\text { Mass }\end{array}$} & \multirow{2}{*}{$\begin{array}{l}\text { Muscle } \\
\text { Strength }\end{array}$} & \multicolumn{2}{|c|}{ Physical Performance } & & & & \\
\hline & & & & Total & Male & Female & & & & $\begin{array}{l}\text { Distance } \\
\text { (m) }\end{array}$ & Gait Speed & Total & Male & Female & \\
\hline \multicolumn{16}{|c|}{ Outpatient Services $(n=4)$} \\
\hline Li et al., 2014 [74] & Chinese & Mainland & $\begin{array}{l}\text { Cross- } \\
\text { sectional }\end{array}$ & 169 & 169 & - & $\begin{array}{c}\text { IWGS } \\
\text { EWGSOP }\end{array}$ & DXA & Dynamometer & 6 & $\begin{array}{l}\text { Usual } \\
\text { Usual }\end{array}$ & $\begin{array}{c}106(62.9) \\
56(33.3)\end{array}$ & $\begin{array}{c}106(62.9) \\
56(33.3)\end{array}$ & - & Moderate \\
\hline Wang et al., 2016 [75] & Chinese & Mainland & $\begin{array}{l}\text { Cross- } \\
\text { sectional }\end{array}$ & 410 & - & - & EWGSOP & DXA & Dynamometer & 6 & Usual & 80 (19.5) & - & - & Moderate \\
\hline Fung et al., 2019 [42] & English & Singapore & $\begin{array}{c}\text { Cross- } \\
\text { sectional }\end{array}$ & 266 & - & - & AWGS & BIA & Dynamometer & 6 & Usual & $70(26.3)$ & - & - & low \\
\hline Wang et al., 2019 [76] & Chinese & Mainland & $\begin{array}{c}\text { Cross- } \\
\text { sectional }\end{array}$ & 430 & 191 & 239 & EWGSOP & BIA & Dynamometer & 6 & Usual & $95(22.1)$ & $32(16.8)$ & $63(26.4)$ & Moderate \\
\hline \multicolumn{16}{|c|}{ Nursing Home $(n=5)$} \\
\hline Hsu et al., 2014 [77] & English & Taiwan & $\begin{array}{l}\text { Cross- } \\
\text { sectional }\end{array}$ & 353 & 353 & - & EWGSOP & BIA & Dynamometer & 6 & Usual & $109(30.9)$ & $109(30.9)$ & - & Moderate \\
\hline Wu et al., 2017 [43] & Chinese & Mainland & $\begin{array}{l}\text { Cross- } \\
\text { sectional }\end{array}$ & 786 & 320 & 466 & EWGSOP & BIA & Dynamometer & 4 & - & $199(25.3)$ & $64(20.0)$ & $135(29.0)$ & Moderate \\
\hline Liao 2018 [78] & Chinese & Mainland & $\begin{array}{l}\text { Cross- } \\
\text { sectional }\end{array}$ & 225 & 63 & 162 & AWGS & BIA & Dynamometer & 6 & Usual & $86(38.2)$ & $26(41.3)$ & $60(37.0)$ & Moderate \\
\hline Zeng et al., 2018 [79] & English & Mainland & $\begin{array}{l}\text { Cross- } \\
\text { sectional }\end{array}$ & 277 & 83 & 194 & FNIH & BIA & Dynamometer & 4 & Usual & $87(31.4)$ & $19(22.9)$ & $68(35.1)$ & Moderate \\
\hline Yang et al., 2019 [80] & English & Mainland & $\begin{array}{c}\text { Cross- } \\
\text { sectional }\end{array}$ & 316 & 112 & 204 & AWGS & BIA & Dynamometer & 4 & - & $91(28.8)$ & $34(30.4)$ & $57(27.9)$ & Moderate \\
\hline \multicolumn{16}{|c|}{ Mixed Settings: Communities and Nursing Homes $(n=2)$} \\
\hline Chen 2018 [81] & Chinese & Mainland & $\begin{array}{l}\text { Cross- } \\
\text { sectional }\end{array}$ & 158 & 43 & 115 & AWGS & BIA & Dynamometer & 6 & Usual & $34(21.5)$ & $5(11.4)$ & $29(25.4)$ & Moderate \\
\hline Yang 2018 [44] & Chinese & Mainland & $\begin{array}{c}\text { Cross- } \\
\text { sectional }\end{array}$ & 316 & 112 & 204 & AWGS & BIA & Dynamometer & 4 & Usual & $91(28.8)$ & $34(30.4)$ & $57(27.9)$ & Low \\
\hline \multicolumn{16}{|c|}{ Mixed Settings: Hospital and Outpatient Services $(n=5)$} \\
\hline Feng 2016 [82] & Chinese & Mainland & $\begin{array}{c}\text { Cross- } \\
\text { sectional }\end{array}$ & 330 & 157 & 173 & AWGS & BIA & Dynamometer & 4 & Maximal & 35 (10.6) & $21(13.4)$ & $14(8.1)$ & Moderate \\
\hline Ma 2017 [83] & Chinese & Mainland & $\begin{array}{c}\text { Cross- } \\
\text { sectional }\end{array}$ & 764 & 550 & 214 & AWGS & BIA & Dynamometer & 4 & Usual & 138 (18.1) & $82(14.9)$ & $56(26.2)$ & Moderate \\
\hline Zhou et al., 2018 [84] & Chinese & Mainland & $\begin{array}{l}\text { Cross- } \\
\text { sectional }\end{array}$ & 163 & 100 & 63 & IWGS & DXA & Dynamometer & 3 & Maximal & $26(16.0)$ & - & - & Moderate \\
\hline Zhang et al., 2019 [85] & Chinese & Mainland & $\begin{array}{l}\text { Cross- } \\
\text { sectional }\end{array}$ & 223 & - & - & AWGS & BIA & Dynamometer & 6 & Usual & $49(22.0)$ & - & - & Moderate \\
\hline Yang 2019 [86] & Chinese & Mainland & $\begin{array}{c}\text { Cross- } \\
\text { sectional }\end{array}$ & 102 & 51 & 51 & AWGS & BIA & Dynamometer & 4 & Maximal & $17(16.0)$ & - & - & Moderate \\
\hline
\end{tabular}

${ }^{+}$: This study provided sarcopenia prevalence for older adults from communities and clinical settings separately. 


\subsection{Prevalence in Older Men}

In the 46 studies that reported the prevalence of sarcopenia in older men, the overall prevalence was $18 \%$ (95\% CI: 15.7 to $20.4 \%, \mathrm{I}^{2}=95.2 \%$ ). For participants from different settings, the pooled prevalence rates were $12.9 \%\left(95 \%\right.$ CI: 10.7 to $\left.15.1 \%, \mathrm{I}^{2}=93.7 \%\right)$ in community-dwelling older men $(n=27), 29.7 \%$ (95\% CI: 18.4 to $\left.41.1 \%, \mathrm{I}^{2}=95.5 \%\right)$ for hospitalized older men $(n=9)$, and $26.3 \%$ (95\% CI: 19.1 to $33.4 \%, \mathrm{I}^{2}=83.7 \%$ ) in nursinghome residents $(n=5)$. We did not pool the prevalence for outpatients due to an insufficient number of studies $(n=2)$. Substantial heterogeneity in prevalence estimates was found across the studies, with all of the I-square values being greater than $80 \%$.

\subsection{Prevalence in Older Women}

In the 44 studies that reported the prevalence of sarcopenia in older women, the overall prevalence was $16.4 \%$ (95\% CI: 14.1 to $18.8 \%, \mathrm{I}^{2}=97.3 \%$ ). For participants from different settings, the prevalence was $11.2 \%$ (95\% CI: 8.9 to $\left.13.4 \%, \mathrm{I}^{2}=97.1 \%\right)$ in community-dwelling older women, $(n=27), 23.0 \%$ (95\% CI: 17.1 to $28.8 \%, \mathrm{I}^{2}=80.9 \%$ ) for hospitalized older women $(n=9)$, and $33.7 \%$ (95\% CI: 27.2 to $40.1 \%, \mathrm{I}^{2}=78.4 \%$ ) for those from nursing homes $(n=4)$. Because only one study reported the prevalence for female outpatients, we did not perform meta-analysis for outpatients. Similarly, considerable heterogeneity was found across studies.

\subsection{Meta-Regression Analysis}

We performed a multivariable meta-regression to explore the potential sources of the considerable heterogeneity in the estimates of sarcopenia prevalence across studies. The results of the regression indicated that, irrespective of gender, the hospitalized patients and nursing-home residents had a higher prevalence of sarcopenia than community-dwelling older adults. For older men, the prevalence rate was lower when muscle mass was assessed using the BIA method rather than the DXA method. Furthermore, those from mainland China appeared to have a higher prevalence of sarcopenia compared with those from Hong Kong or Taiwan. For older women, only the participants from different settings showed a significant association with the prevalence of sarcopenia (Table 2).

Table 2. Multivariable meta-regression.

\begin{tabular}{|c|c|c|c|c|c|c|}
\hline \multirow{2}{*}{ Covariates } & \multicolumn{3}{|c|}{ Males $(n=43)$} & \multicolumn{3}{|c|}{ Females $(n=41)$} \\
\hline & $\operatorname{Exp}(\beta)$ & $95 \%$ CI & $p$-Value & $\operatorname{Exp}(\beta)$ & $95 \%$ CI & $p$-Value \\
\hline \multicolumn{7}{|l|}{ Populations } \\
\hline Community-dwelling (ref) & 1.00 & & & 1.00 & & \\
\hline Outpatients & 1.29 & $(0.52,3.17)$ & 0.570 & 2.28 & $(0.67,7.73)$ & 0.180 \\
\hline Hospitalized people & 1.69 & $(1.01,2.86)$ & 0.047 & 2.10 & $(1.17,3.78)$ & 0.015 \\
\hline Nursing-home residents & 2.50 & $(1.35,4.66)$ & 0.005 & 2.73 & $(1.38,5.38)$ & 0.005 \\
\hline \multicolumn{7}{|l|}{ Diagnosis criteria } \\
\hline AWGS (ref) & 1.00 & & & 1.00 & & \\
\hline EWGSOP & 1.23 & $(0.67,2.27)$ & 0.490 & 0.92 & $(0.39,2.15)$ & 0.840 \\
\hline \multicolumn{7}{|l|}{ Assessment of muscle mass } \\
\hline DXA (ref) & 1.00 & & & 1.00 & & \\
\hline BIA & 0.58 & $(0.35,0.98)$ & 0.044 & 1.17 & $(0.60,2.29)$ & 0.640 \\
\hline \multicolumn{7}{|l|}{ Area } \\
\hline Mainland (ref) & 1.00 & & & 1.00 & & \\
\hline Out of mainland & 0.47 & $(0.22,0.98)$ & 0.045 & 0.51 & $(0.18,1.43)$ & 0.190 \\
\hline \multicolumn{7}{|l|}{ Walk distance } \\
\hline 6 m (ref) & 1.00 & & & 1.00 & & \\
\hline $4 \mathrm{~m}$ & 0.84 & $(0.53,1.32)$ & 0.440 & 1.12 & $(0.68,1.83)$ & 0.650 \\
\hline Others & 0.81 & $(0.34,1.93)$ & 0.630 & 0.73 & $(0.24,2.25)$ & 0.580 \\
\hline
\end{tabular}




\section{Discussion}

This meta-analysis provided a comprehensive picture of the prevalence of sarcopenia in Chinese older adults. It filled the knowledge gap by extending the existing reviews of sarcopenia prevalence in Chinese older adults from communities to other settings (clinical settings and nursing homes) as well. We also systematically investigated factors that contributed to the significant heterogeneity in pooled prevalence estimates using multivariable meta-regression. Our findings contribute to the epidemiological literature on sarcopenia in Chinese older adults, and can provide a starting point for future research and efforts in sarcopenia prevention among Chinese populations.

For community-dwelling Chinese older adults, we obtained similar estimates of prevalence $(12.9 \%$ in men vs. $11.2 \%$ in women) compared with previous reports ( $11 \%$ in men vs. $10 \%$ in women; $14 \%$ in men vs. $9 \%$ in women) [24,25]. When compared with the prevalence in Europeans (13\% in men vs. $14 \%$ in women), our estimates were comparable with them [18]. However, Papadopoulou et al. [18] found non-Asian groups seemed more prone to sarcopenia than Asian groups. The difference in ethnic characteristics, body size, and dietary regimes etc., between Asians and non-Asians might be possible reasons for the potential disparity in the sarcopenia prevalence. As for gender difference, our result was similar to the previous evidence that higher prevalence of sarcopenia was shown in Chinese community-dwelling older males than females $[15,22,25]$. However, in western countries, the evidence of gender difference was controversial depending on the EWGSOP cut-off values applied $[13,87]$. For hospitalized older adults, we found the prevalence to be $29.7 \%$ in men and $23.0 \%$ in women. To our knowledge, this is the first systematic review to pool the prevalence of sarcopenia for Chinese older adults who are hospitalized. Our findings revealed a relatively higher prevalence in this group compared with that from communities. Compared with the pooled estimates of hospitalized older adults mainly from Europe ( $23 \%$ in men and $24 \%$ in women), our findings were higher in hospitalized men and comparable in hospitalized women [18]. For outpatients, considering that Cochran's Q is not very informative and tends to be biased when the number of studies is small, we did not perform meta-analysis for this group [88]. For those residing in nursing homes, similarly, no meta-analysis synthesized the prevalence of sarcopenia in this Chinese group before. Shen et al. [28] conducted a systematic review pooling the prevalence of sarcopenia in nursing-home populations from 16 studies. However, they only included one original study that targeted the Chinese population [28]. Compared with Shen et al.'s results (43\% in men vs. $46 \%$ in women), we obtained lower pooled estimates ( $26.3 \%$ in men vs. $33.7 \%$ in women) [28]. Nevertheless, the above evidence suggested the high risk of prevalent sarcopenia in nursing-home residents. Institutionalized populations may be more prone to malnutrition $[89,90]$. A recent cross-sectional study found that malnutrition and physical frailty were highly prevalent among institutionalized older residents and malnutrition was associated with an increased risk of physical frailty among institutionalized Chinese older adults [89]. Hence, nutrition and exercise intervention should be promoted to the institutionalized population to prevent or reverse muscle function for this population.

Multivariable meta-regression indicated that the populations being from different settings was a significant factor contributing to the variation in the pooled prevalence estimates for both genders. Compared with community-dwelling older residents, those who were hospitalized were at 1.69 to 2.10 times the risk of prevalent sarcopenia, and nursing-home residents had 2.50 to 2.73 times the risk. The much higher prevalence rate of sarcopenia in older adults from hospitals and nursing homes was also reported by Papadopoulou and colleagues [18]. However, while Papadopoulou and colleagues reported variation in the pooled prevalence estimates for older adults from different settings, they did not perform meta-regression to quantify it. Our study provided the evidence regarding how much the risk of prevalence increased for older adults who were hospitalized or residing in a nursing home compared with their community-dwelling counterparts. Our findings suggest that hospitalized older adults and those living in nursing homes are particularly vulnerable to this muscle disorder. Therefore, these populations should be 
prioritized in sarcopenia-screening and early-prevention efforts. Furthermore, intervention studies of sarcopenia targeting hospitalized older adults and those in nursing homes are warranted to inform evidence-based prevention and management efforts such as exercise and/or nutrition interventions for these susceptible groups. Meta-regression did not show significant difference in the prevalence estimate between the community-dwelling and outpatient groups for both genders. However, there were a small number of studies targeting outpatients, which might reduce the statistical power. Future research could further examine the risk of prevalent sarcopenia in this group of older adults.

We found the prevalence estimate in older men varied depending on the method used to assess muscle mass. Specifically, compared with the DXA method, the risk of prevalence was lower (OR: $0.58,95 \%$ CI: 0.35 to 0.98 ) when using the BIA method. However, no such difference was detected in older women. There is some evidence that the BIA method tends to yield higher estimates of muscle mass, and therefore lower estimates of the prevalence of sarcopenia than the DXA method [91]. Wu and Li [24] synthesized the prevalence estimates from 16 original studies targeting community-dwelling Chinese older adults and obtained similar prevalence estimates irrespective of muscle mass assessments (DXA: 13\%, BIA: 12\%). However, they did not perform a meta-regression to further examine whether different muscle mass assessments could result in significantly different prevalence estimates after adjusting other covariates. In fact, the measurement of muscle mass remains a challenge in primary care settings; and, while DXA is the standard and more accurate method, BIA is preferred in primary care settings and research because it is more available, easier to use, and less expensive [92]. There are different types of equipment and frequencies of BIA. Based on previous study, BIA with a multifrequency device showed better agreement with the DXA method than that with other devices when assessing appendicular skeletal muscle mass [51]. The updated AWGS consensus in 2019 also recommended the use of multifrequency BIA [93]. However, the "model" of BIA measurement was not always stated explicitly in previous studies. Therefore, in future research, if the BIA method is used to assess muscle mass, the multifrequency type should be used and clearly documented.

Our study also found the risk of prevalent sarcopenia in older Chinese men living outside the mainland, specifically, in Hong Kong and Taiwan, to be nearly half that of their counterparts living on the mainland (OR: $0.47,95 \%$ CI: 0.23 to 0.98 ). However, this difference was not significant in the case of older Chinese women. Regional variation in the prevalence of sarcopenia among community-dwelling Chinese older adults was also reported in a previous systematic review, with 17\% in mainland and 6\% in Hong Kong and Taiwan [24]. However, that review did not conduct meta-regression to quantify the regional variation in the prevalence of sarcopenia. Possible explanations for the variation between mainland Chinese and those living outside the mainland might be attributed to the different levels of economic development, different healthcare and dietary regimes, etc. [94]. Future studies could further explore the reasons why the prevalence of sarcopenia among older adults living outside the mainland is lower than for those in mainland China. Furthermore, as mainland China has a huge area with diverse geographic and climate variations, the prevalence of sarcopenia might also vary across different geographic locations. Until now, no study has investigated the epidemiological characteristics of sarcopenia among different geographic areas within China. Therefore, we further divided the area into 4 regions (south and east of mainland, west of mainland, north of mainland, and outside of mainland) and ran the univariable mate-regression. Findings showed that significant difference in the prevalence of sarcopenia was only presented between mainland areas and outside of the mainland; there was no difference among different geographic locations within mainland China in both genders (Supplementary Materials, Table S3). Therefore, we only divided the area into two regions (mainland and outside of the mainland) in the main analysis.

Previous observational studies found that older age was significantly associated with sarcopenia and severe sarcopenia in Chinese older adults [46,50]. However, less than half of included studies reported the gender-specific age (Supplementary Materials, Table S4). Therefore, our main results did not consider the age in the multivariable meta-regression. 
Instead, we conducted a supplementary analysis to fit the univariable meta-regression for age (details are presented in Supplementary Materials, Table S5). Findings indicated that the age group of 80 years and over showed a higher prevalence of sarcopenia compared with that of 60 to 70 years, which is consistent with current knowledge that the advanced age is an important risk factor of sarcopenia [50]. Body mass index (BMI) was also reported to be associated with the prevalence of sarcopenia in some literature, with a higher BMI relating to the lower prevalence of sarcopenia $[46,50]$. In this systematic review, BMI values reported in articles were all above $23 \mathrm{~kg} / \mathrm{m}^{2}$ for both genders (Supplementary Materials, Table S4). We did not include BMI in the meta-regression, considering that the comparison between overweight and obesity might be less meaningful. For other possible factors which might influence the variance of prevalence reported, such as rural-urban regions, comorbidities, and others (e.g., type of dynamometer, protocol of gait speed test), due to limited information reported in the articles, we could not analyze them through meta-regression.

The present study was subject to several limitations. Firstly, a few of the included studies reported the prevalence by different age groups, so we could not pool the prevalence rate by age subgroup. However, age is a well-established factor of sarcopenia. The lower bound of age for participants in the studies included in this review ranged considerably, from 60 to 80 years. Therefore, age may be a significant source of the substantial heterogeneity that we found across studies. Future epidemiological research of sarcopenia is encouraged to report age-specific prevalence. Secondly, due to the small number of studies which reported the prevalence of sarcopenia for outpatients, we were unable to obtain a pooled prevalence rate for this group. Thirdly, we did not include all of the possible covariates in our meta-regression analysis to investigate the source of considerable variations in sarcopenia prevalence. For example, all of the included studies used dynamometers to assess muscle strength, but we had insufficient information to investigate the potential influence of the type of dynamometer used on the prevalence rate. We were likewise unable to analyze the influence of different measurement protocols used for the gait speed tests due to insufficient information. Finally, our results should be interpreted with caution because most of the included studies had a moderate risk of bias and significant heterogeneity.

\section{Conclusions}

This meta-analysis and meta-regression provides a comprehensive synthesis of sarcopenia prevalence in current literature targeting Chinese older populations not only from communities, but also from clinical settings and nursing homes. This is, to our knowledge, the first study to report pooled prevalence in Chinese older populations from clinical settings and nursing homes, and to examine the impact of populations from different settings, diagnostic criteria, muscle mass assessments, areas and walk distance of gait speed test on the prevalence estimate of sarcopenia via meta-regression analysis. Despite the variations in prevalence estimate across studies, this study revealed a certain proportion of Chinese older adults suffering from sarcopenia, especially for those who were hospitalized and residing in nursing homes. Considering the accelerating pace of aging, efforts should be made to implement early screening and lifestyle interventions such as nutrition and physical activity promotion to prevent this increasingly widespread age-related geriatric syndrome, especially for vulnerable groups.

Supplementary Materials: The following are available online at https:/ /www.mdpi.com/article/10 .3390/nu13051441/s1, S1: Search strategy; Table S2 Critical Appraisal of Study Quality; Table S3 Univariable meta-regression for regions; Table S4 Age and BMI reported in studies; Table S5 Univariable meta-regression for age.

Author Contributions: Conceptualization, P.-H.C., Z.C., and M.H.; acquisition of data, Z.C. and W.-Y.L.; formal analysis, Z.C. and P.-H.C.; writing-original draft preparation, Z.C.; writing-review and editing, P.-H.C., M.H., Z.C., and W.-Y.L., supervision, P.-H.C. All authors have read and agreed to the published version of the manuscript. 
Funding: This research received no external funding.

Institutional Review Board Statement: Not applicable.

Informed Consent Statement: Not applicable.

Data Availability Statement: Data sharing not applicable.

Conflicts of Interest: The authors declare no conflict of interest.

\section{References}

1. WHO. Ageing and Health. Available online: https://www.who.int/news-room/fact-sheets/detail/ageing-and-health (accessed on 13 May 2020).

2. National Bureau of Statistics. 2019 China Statistical Yearbook. Available online: http://www.stats.gov.cn/tjsj/ndsj/2019/indexeh. htm (accessed on 17 August 2020).

3. Larsson, L.; Degens, H.; Li, M.; Salviati, L.; Lee, Y.I.; Thompson, W.; Kirkland, J.L.; Sandri, M. Sarcopenia: Aging-related loss of muscle mass and function. Physiol. Rev. 2019, 99, 427-511. [CrossRef] [PubMed]

4. Sakuma, K.; Yamaguchi, A. Sarcopenic obesity and endocrinal adaptation with age. Int. J. Endocrinol. 2013, $2013,204164$. [CrossRef] [PubMed]

5. Santilli, V.; Bernetti, A.; Mangone, M.; Paoloni, M. Clinical definition of sarcopenia. Clin. Cases Miner. Bone Metab. 2014, 11, 177-180. [CrossRef] [PubMed]

6. Anton, S.D.; Hida, A.; Mankowski, R.; Layne, A.; Solberg, L.M.; Mainous, A.G.; Buford, T. Nutrition and Exercise in Sarcopenia. Curr. Protein Pept. Sci. 2018, 19, 649-667. [CrossRef]

7. Beckwée, D.; Delaere, A.; Aelbrecht, S.; Baert, V.; Beaudart, C.; Bruyere, O.; de Saint-Hubert, M.; Bautmans, I. Exercise Interventions for the Prevention and Treatment of Sarcopenia. A Systematic Umbrella Review. J. Nutr. Health Aging 2019, 23, 494-502. [CrossRef]

8. Bloom, I.; Shand, C.; Cooper, C.; Robinson, S.; Baird, J. Diet Quality and Sarcopenia in Older Adults: A Systematic Review. Nutrients 2018, 10, 308. [CrossRef]

9. Tessier, A.J.; Chevalier, S. An Update on Protein, Leucine, Omega-3 Fatty Acids, and Vitamin D in the Prevention and Treatment of Sarcopenia and Functional Decline. Nutrients 2018, 10, 1099. [CrossRef]

10. Beaudart, C.; Zaaria, M.; Pasleau, F.; Reginster, J.-Y.; Bruyère, O. Health outcomes of sarcopenia: A systematic review and meta-analysis. PLoS ONE 2017, 12, e0169548. [CrossRef]

11. Pamoukdjian, F.; Bouillet, T.; Lévy, V.; Soussan, M.; Zelek, L.; Paillaud, E. Prevalence and predictive value of pre-therapeutic sarcopenia in cancer patients: A systematic review. Clin. Nutr. 2018, 37, 1101-1113. [CrossRef]

12. Chen, L.K.; Liu, L.K.; Woo, J.; Assantachai, P.; Auyeung, T.W.; Bahyah, K.S.; Chou, M.Y.; Chen, L.Y.; Hsu, P.S.; Krairit, O.; et al. Sarcopenia in Asia: Consensus report of the Asian Working Group for Sarcopenia. J. Am. Med. Dir. Assoc. 2014, 15, 95-101. [CrossRef]

13. Fielding, R.A.; Vellas, B.; Evans, W.J.; Bhasin, S.; Morley, J.E.; Newman, A.B.; Abellan van Kan, G.; Andrieu, S.; Bauer, J.; Breuille, D.; et al. Sarcopenia: An undiagnosed condition in older adults. Current consensus definition: Prevalence, etiology, and consequences. International working group on sarcopenia. J. Am. Med. Dir. Assoc. 2011, 12, 249-256. [CrossRef]

14. Studenski, S.A.; Peters, K.W.; Alley, D.E.; Cawthon, P.M.; McLean, R.R.; Harris, T.B.; Ferrucci, L.; Guralnik, J.M.; Fragala, M.S.; Kenny, A.M.; et al. The FNIH sarcopenia project: Rationale, study description, conference recommendations, and final estimates. J. Gerontol. A Biol. Sci. Med. Sci. 2014, 69, 547-558. [CrossRef] [PubMed]

15. Liu, X.; Hou, L.; Xia, X.; Liu, Y.; Zuo, Z.; Zhang, Y.; Zhao, W.; Hao, Q.; Yue, J.; Dong, B. Prevalence of sarcopenia in multi ethnics adults and the association with cognitive impairment: Findings from West-China health and aging trend study. BMC Geriatr. 2020, 20, 63. [CrossRef]

16. Beaudart, C.; Reginster, J.Y.; Slomian, J.; Buckinx, F.; Dardenne, N.; Quabron, A.; Slangen, C.; Gillain, S.; Petermans, J.; Bruyère, O. Estimation of sarcopenia prevalence using various assessment tools. Exp. Gerontol. 2015, 61, 31-37. [CrossRef]

17. Bruyère, O.; Beaudart, C.; Reginster, J.Y.; Buckinx, F.; Schoene, D.; Hirani, V.; Cooper, C.; Kanis, J.A.; Rizzoli, R.; McCloskey, E.; et al. Assessment of muscle mass, muscle strength and physical performance in clinical practice: An international survey. Eur. Geriatr. Med. 2016, 7, 243-246. [CrossRef]

18. Papadopoulou, S.K.; Tsintavis, P.; Potsaki, P.; Papandreou, D. Differences in the Prevalence of Sarcopenia in Community-Dwelling, Nursing Home and Hospitalized Individuals. A Systematic Review and Meta-Analysis. J. Nutr. Health Aging 2020, $24,83-90$. [CrossRef] [PubMed]

19. Chan, R.; Leung, J.; Woo, J. A prospective cohort study to examine the association between dietary patterns and sarcopenia in Chinese community-dwelling older people in Hong Kong. J. Am. Med. Dir. Assoc. 2016, 17, 336-342. [CrossRef]

20. Huang, C.Y.; Hwang, A.C.; Liu, L.K.; Lee, W.J.; Chen, L.Y.; Peng, L.N.; Lin, M.H.; Chen, L.K. Association of dynapenia, sarcopenia, and cognitive impairment among community-dwelling older Taiwanese. Rejuvenation Res. 2016, 19, 71-78. [CrossRef]

21. Chen, X.; Guo, J.; Han, P.; Fu, L.; Jia, L.; Yu, H.; Yu, X.; Hou, L.; Wang, L.; Zhang, W.; et al. Twelve-month incidence of depressive symptoms in suburb-dwelling Chinese older adults: Role of sarcopenia. J. Am. Med. Dir. Assoc. 2019, 20, 64-69. [CrossRef] 
22. Du, Y.; Wang, X.; Xie, H.; Zheng, S.; Wu, X.; Zhu, X.; Zhang, X.; Xue, S.; Li, H.; Hong, W.; et al. Sex differences in the prevalence and adverse outcomes of sarcopenia and sarcopenic obesity in community dwelling elderly in East China using the AWGS criteria. BMC Endocr. Disord. 2019, 19, 109. [CrossRef] [PubMed]

23. Wang, H.; Hai, S.; Liu, Y.; Cao, L.; Liu, Y.; Liu, P.; Zhou, J.; Yang, Y.; Dong, B. Association between depressive symptoms and sarcopenia in older Chinese community-dwelling individuals. Clin. Interv. Aging 2018, 13, 1605-1611. [CrossRef]

24. Wu, L.J.; Li, J.X. Prevalence of sarcopenia in the community-dwelling elder people in China: A systematic review and metaanalysis. Modern Prev. Med. 2019, 46, 18. (In Chinese)

25. Tian, S.; Xu, Y.; Han, F. Prevalence of sarcopenia in the community-dwelling, elderly Chinese population: A systematic review and meta-analysis. Lancet 2017, 390, S35. [CrossRef]

26. Moher, D.; Liberati, A.; Tetzlaff, J.; Altman, D.G. Preferred reporting items for systematic reviews and meta-analyses: The PRISMA statement. PLoS Med. 2009, 6, e1000097. [CrossRef]

27. Hoy, D.; Brooks, P.; Woolf, A.; Blyth, F.; March, L.; Bain, C.; Baker, P.; Smith, E.; Buchbinder, R. Assessing risk of bias in prevalence studies: Modification of an existing tool and evidence of interrater agreement. J. Clin. Epidemiol. 2012, 65, 934-939. [CrossRef] [PubMed]

28. Shen, Y.; Chen, J.; Chen, X.; Hou, L.; Lin, X.; Yang, M. Prevalence and Associated Factors of Sarcopenia in Nursing Home Residents: A Systematic Review and Meta-analysis. J. Am. Med. Dir. Assoc. 2019, 20, 5-13. [CrossRef] [PubMed]

29. Mogire, R.M.; Mutua, A.; Kimita, W.; Kamau, A.; Bejon, P.; Pettifor, J.M.; Adeyemo, A.; Williams, T.N.; Atkinson, S.H. Prevalence of vitamin D deficiency in Africa: A systematic review and meta-analysis. Lancet Glob. Health 2020, 8, e134-e142. [CrossRef]

30. Higgins, J.; Thomas, J.; Chandler, J.; Cumpston, M.; Li, T.; Page, M.; Welch, V. Cochrane Handbook for Systematic Reviews of Interventions, 2nd ed.; John Wiley \& Sons: Chichester, UK, 2019.

31. StataCorp. Stata Statistical Software: Release 15; StataCorp LP: College Station, TX, USA, 2017.

32. Nyaga, V.N.; Arbyn, M.; Aerts, M. Metaprop: A Stata command to perform meta-analysis of binomial data. Arch. Public Health 2014, 72, 39. [CrossRef]

33. Higgins, J.P.; Thompson, S.G.; Deeks, J.J.; Altman, D.G. Measuring inconsistency in meta-analyses. BMJ 2003, 327, 557-560. [CrossRef]

34. Easterbrook, P.J.; Berlin, J.A.; Gopalan, R.; Matthews, D.R. Publication bias in clinical research. Lancet 1991, 337, 867-872. [CrossRef]

35. Meng, N.H.; Li, C.I.; Liu, C.S.; Lin, C.H.; Lin, W.Y.; Chang, C.K.; Li, T.C.; Lin, C.C. Comparison of height- and weight-adjusted sarcopenia in a Taiwanese metropolitan older population. Geriatr. Gerontol. Int. 2015, 15, 45-53. [CrossRef]

36. Wen, X.; An, P.; Chen, W.C.; Lv, Y.; Fu, Q. Comparisions of sarcopenia prevalence based on different disgnostic criteria in Chinese older adults. J. Nutr. Health Aging 2015, 19, 342-347. [CrossRef]

37. Xia, Z.W.; Meng, L.C.; Man, Q.Q.; Li, L.X.; Song, P.K.; Li, Y.Q.; Gao, Y.X.; Jia, S.S.; Zhang, J. Analysis of the dietary factors on sarcopenia in elderly in Beijing. J. Hyg. Res. 2016, 45, 388-393. (In Chinese) [CrossRef]

38. Zhang, Y.; Tan, Y.T.; Huang, X.X.; Zhang, Z.H.; Bai, J.J.; Zhang, M.; Huang, Y.Q.; Chen, J.; Wang, J.F.; Bao, Z.J. Prevalence of Sarcopenia and the Associated Risk Factors in Community Elderly in Shanghai. Geriatr. Health Care 2018, 24, 608-613. (In Chinese)

39. Liu, J.L.; Cai, Y.Y. Investigation of prevalence among community-dwelling older adults in Shanghai. China Health Vis. 2016, 248-249. (In Chinese) [CrossRef]

40. Xu, H.Q.; Shi, J.P.; Shen, C.; Liu, Y.; Liu, J.M.; Zheng, X.Y. Sarcopenia-related features and factors associated with low muscle mass, weak muscle strength, and reduced function in Chinese rural residents: A cross-sectional study. Arch. Osteoporos. 2018, 14, 2. [CrossRef]

41. Wang, Z.T. Correlation Analysis of Coronary Heart Disease and Sarcopenia in Elderly Inpatients. Master's Thesis, Southern Medical University, Guangzhou, China, 2019.

42. Fung, F.Y.; Koh, Y.L.E.; Malhotra, R.; Ostbye, T.; Lee, P.Y.; Shariff Ghazali, S.; Tan, N.C. Prevalence of and factors associated with sarcopenia among multi-ethnic ambulatory older Asians with type 2 diabetes mellitus in a primary care setting. BMC Geriatr. 2019, 19, 1-10. [CrossRef]

43. Wu, Z.L.; Wang, Y.B.; Su, F.Q.; Song, B.; Li, G.; Zhu, H.M. Epidemiological investigation of sarcopenia in the nursing homes in Fengxian District and analysis of their risk factors. Shanghai Med. Pharm. J. 2017, 38, 37-40. (In Chinese)

44. Yang, L.J. Research on Sarcopenia and Its Relative factors in Elderly Population in Suzhou. Master's Thesis, Nanjing Medical University, Suzhou, China, 2018.

45. Meng, P.; Hu, Y.X.; Fan, L.; Zhang, Y.; Zhang, M.X.; Sun, J.; Liu, Y.; Li, M.; Yang, Y.; Wang, L.H.; et al. Sarcopenia and sarcopenic obesity among men aged 80 years and older in Beijing: Prevalence and its association with functional performance. Geriatr. Gerontol. Int. 2014, 14 (Suppl. 1), 29-35. [CrossRef]

46. Wu, C.H.; Chen, K.T.; Hou, M.T.; Chang, Y.F.; Chang, C.S.; Liu, P.Y.; Wu, S.J.; Chiu, C.J.; Jou, I.M.; Chen, C.Y. Prevalence and associated factors of sarcopenia and severe sarcopenia in older Taiwanese living in rural community: The Tianliao Old People study 04. Geriatr. Gerontol. Int. 2014, 14 (Suppl. 1), 69-75. [CrossRef]

47. Zhang, Y.; Hu, Y.X.; Fan, L.; Zhang, M.X.; Sun, J.; Han, X.Q.; Ma, X.N.; Dong, H.Y.; Li, M. Relationship between site-specific loss of bodr skeletal muscle mass and gait performance in very old men in Beijing. Chin. J. Health Care Med. 2014, 16, 421-425. (In Chinese) [CrossRef] 
48. Wang, Y.J.; Wang, Y.; Zhan, J.K.; Tang, Z.Y.; He, J.Y.; Tan, P.; Deng, H.Q.; Huang, W.; Liu, Y.S. Sarco-Osteoporosis: Prevalence and Association with Frailty in Chinese Community-Dwelling Older Adults. Int. J. Endocrinol. 2015, 2015, 482940. [CrossRef]

49. Han, D.S.; Chang, K.V.; Li, C.M.; Lin, Y.H.; Kao, T.W.; Tsai, K.S.; Wang, T.G.; Yang, W.S. Skeletal muscle mass adjusted by height correlated better with muscular functions than that adjusted by body weight in defining sarcopenia. Sci. Rep. 2016, 6, 19457. [CrossRef]

50. Han, P.; Kang, L.; Guo, Q.; Wang, J.; Zhang, W.; Shen, S.; Wang, X.; Dong, R.; Ma, Y.; Shi, Y.; et al. Prevalence and Factors Associated With Sarcopenia in Suburb-dwelling Older Chinese Using the Asian Working Group for Sarcopenia Definition. J. Gerontol. Ser. A Biol. Sci. Med. Sci. 2016, 71, 529-535. [CrossRef] [PubMed]

51. Wang, H.; Hai, S.; Cao, L.; Zhou, J.; Liu, P.; Dong, B.R. Estimation of prevalence of sarcopenia by using a new bioelectrical impedance analysis in Chinese community-dwelling elderly people. BMC Geriatr. 2016, 16, 216. [CrossRef]

52. Wang, T.; Feng, X.; Zhou, J.; Gong, H.; Xia, S.; Wei, Q.; Hu, X.; Tao, R.; Li, L.; Qian, F.; et al. Type 2 diabetes mellitus is associated with increased risks of sarcopenia and pre-sarcopenia in Chinese elderly. Sci. Rep. 2016, 6, 38937. [CrossRef]

53. Fang, Y.; Pan, L.; Chen, L.; Chen, J.Y.; Peng, Y.D.; Gu, W.S.; You, L. Sarcopenia screening for older women with low body-weight and low handgrip strength is more urgently. Chin. J. Endocrinol. Metab. 2017, 33, 1043-1046. (In Chinese)

54. Hai, S.; Cao, L.; Wang, H.; Zhou, J.; Liu, P.; Yang, Y.; Hao, Q.; Dong, B. Association between sarcopenia and nutritional status and physical activity among community-dwelling Chinese adults aged 60 years and older. Geriatr. Gerontol. Int. 2017, 17, 1959-1966. [CrossRef]

55. Hua, C.; Chen, G.L.; Wen, X.L.; Liu, C.; Zhang, J. HMB intervention of muscle loss in community-dwelling elders with malnutrition. Electron. J. Metab. Nutr. Cancer 2017, 4, 72-77. (In Chinese) [CrossRef]

56. Meng, L.; Shi, J.; Zou, C.S.; Tan, X.; Zhou, B.Y.; Duan, C.B.; Shi, H.; Xi, H. Correlation of frailty severity with muscle mass and physical function in Chinese older adults:preliminary findings. Chin. J. Geriatr. 2017, 36, 1313-1317. (In Chinese) [CrossRef]

57. Chu, X.J. Osteopenia and Sarcopenia in Chinese Elderly. Master's Thesis, Jiangsu University, Suzhou, China, 2018.

58. Yang, M.; Hu, X.; Xie, L.; Zhang, L.; Zhou, J.; Lin, J.; Wang, Y.; Li, Y.; Han, Z.; Zhang, D.; et al. Validation of the Chinese version of the Mini Sarcopenia Risk Assessment questionnaire in community-dwelling older adults. Medicine 2018, 97, e12426. [CrossRef]

59. Liu, L.L. Prevalence and Risk Factors of Sarcopenia in Meddle-Aged and Elderly Population in Urban Area of Chongqing, China. Master's Thesis, Chongqing Medical University, Chongqing, China, 2019.

60. Wang, H.; Hai, S.; Liu, Y.X.; Cao, L.; Liu, Y.; Liu, P.; Yang, Y.; Dong, B.R. Associations between Sarcopenic Obesity and Cognitive Impairment in Elderly Chinese Community-Dwelling Individuals. J. Nutr. Health Aging 2019, 23, 14-20. [CrossRef]

61. Zhang, L.; Guo, Q.; Feng, B.L.; Wang, C.Y.; Han, P.P.; Hu, J.; Sun, X.D.; Zeng, W.F.; Zheng, Z.X.; Li, H.S.; et al. A Cross-Sectional Study of the Association between Arterial Stiffness and Sarcopenia in Chinese Community-Dwelling Elderly Using the Asian Working Group for Sarcopenia Criteria. J. Nutr. Health Aging 2019, 23, 195-201. [CrossRef]

62. Rong, Y.D.; Bian, A.L.; Hu, H.Y.; Ma, Y.; Zhou, X.Z. A cross-sectional study of the relationships between different components of sarcopenia and brachial ankle pulse wave velocity in community-dwelling elderly. BMC Geriatr. 2020, 20, 115. [CrossRef] [PubMed]

63. Xu, W.; Chen, T.; Cai, Y.; Hu, Y.; Fan, L.; Wu, C. Sarcopenia in Community-Dwelling Oldest Old Is Associated with Disability and Poor Physical Function. J. Nutr. Health Aging 2020, 24, 339-345. [CrossRef]

64. Yang, L.; Yao, X.; Shen, J.; Sun, G.; Sun, Q.; Tian, X.; Li, X.; Li, X.; Ye, L.; Zhang, Z.; et al. Comparison of revised EWGSOP criteria and four other diagnostic criteria of sarcopenia in Chinese community-dwelling elderly residents. Exp. Gerontol. 2020, 130, 110798. [CrossRef] [PubMed]

65. Cui, M.Z. Clinical Characteristics of Sarcopenia among Patients with Type2 Diabetes. Master's Thesis, Jilin University, Changchun, China, 2018.

66. Zhai, Y.; Xiao, Q.; Miao, J. The Relationship between NAFLD and Sarcopenia in Elderly Patients. Can. J. Gastroenterol. Hepatol. 2018, 2018, 5016091. [CrossRef]

67. Chen, Q.; Hao, Q.; Ding, Y.; Dong, B. The Association between Sarcopenia and Prealbumin Levels among Elderly Chinese Inpatients. J. Nutr. Health Aging 2019, 23, 122-127. [CrossRef]

68. Yao, S.H. Prevalence of Sarcopenia and Osteoporosis and Analysis of Their Risk Factors and Correlation in Elderly Hospitalized Patients. Master's Thesis, Jishou University, Jishou, China, 2019.

69. Yi, H.W.; Li, W.L.; Yu, Y.L.; Ma, D.B. The relative factors analysis of malnutrition status and sarcopenia in elderly patients. Electron. J. Metab. Nutr. Cancer 2019, 6. (In Chinese) [CrossRef]

70. Tan, Z.Q. Study on the Influencing Factors of Sarcopenia in Elderly Patients with HFp EF, HFmr EF, and HFr EF. Master's Thesis, Chengdu Medical College, Chengdu, China, 2019.

71. Zhang, N.; Zhu, W.L.; Liu, X.H.; Chen, W.; Zhu, M.L.; Kang, L.; Tian, R. Prevalence and prognostic implications of sarcopenia in older patients with coronary heart disease. J. Geriatr. Cardiol. 2019, 16, 756-763. [CrossRef] [PubMed]

72. Cui, M.; Gang, X.; Wang, G.; Xiao, X.; Li, Z.; Jiang, Z.; Wang, G. A cross-sectional study: Associations between sarcopenia and clinical characteristics of patients with type 2 diabetes. Medicine 2020, 99, e18708. [CrossRef]

73. Wang, L.; Wei, Y.L.; Liu, J.; Wang, J.T. Related factors for sarcopenia in elderly hospitalized patients with chronic diseases. Chin. General. Practic. 2020, 23, 611-616. (In Chinese) [CrossRef] 
74. Li, M.; Hu, Y.X.; Dong, H.Y.; Zhang, Y.; Fan, L.; Zhang, M.X.; Sun, J.; Han, X.Q.; Liu, Y.X.; Ma, X.N. Compare different measurement for prevalence of sarcopenia in a cohort of healthy community-dwelling older men in Beijing area. Chin. J. Health Care Med. 2014, 16, 426-429. (In Chinese) [CrossRef]

75. Wang, R.; Hu, Y.X.; Fan, L.; Cui, H.; Gao, L.G.; Zhang, Y.; Gao, D.W.; Cao, J.; Gong, W.Q. Effect of sarcopenia on the rate of rehospitalization in elderly male patients. Chin. J. Health Care Med. 2016, 18, 106-109. (In Chinese) [CrossRef]

76. Wang, Y.N.; Song, H.L.; Gu, Y.H.; Xu, J.L. Influencing factors of sarcopenia among older adults with type2 diabetes. Prev. Med. 2019, 31, 582-585. (In Chinese) [CrossRef]

77. Hsu, Y.H.; Liang, C.K.; Chou, M.Y.; Liao, M.C.; Lin, Y.T.; Chen, L.K.; Lo, Y.K. Association of cognitive impairment, depressive symptoms and sarcopenia among healthy older men in the veterans retirement community in southern Taiwan: A cross-sectional study. Geriatr. Gerontol. Int. 2014, 14 (Suppl. 1), 102-108. [CrossRef]

78. Liao, S.W. Prevalence and Risk Factors of Sarcopenia among Older Adults Living in Nursing Homes in Chongqing. Master's Thesis, Chongqing Medical University, Chongqing, China, 2018.

79. Zeng, Y.; Hu, X.; Xie, L.; Han, Z.; Zuo, Y.; Yang, M. The Prevalence of Sarcopenia in Chinese Elderly Nursing Home Residents: A Comparison of 4 Diagnostic Criteria. J. Am. Med. Dir. Assoc. 2018, 19, 690-695. [CrossRef] [PubMed]

80. Yang, L.J.; Wu, G.H.; Yang, Y.L.; Wu, Y.H.; Zhang, L.; Wang, M.H.; Mo, L.Y.; Xue, G.; Wang, C.Z.; Weng, X.F. Nutrition, Physical Exercise, and the Prevalence of Sarcopenia in Elderly Residents in Nursing Homes in China. Med. Sci. Monit. 2019, 25, 4390-4399. [CrossRef]

81. Chen, J.M. The Associated Factors of Traditional Chinese Medicine Syndromes in Older Adults with Sarcopenia. Master's Thesis, Beijing University of Chinese Medicine, Beijing, China, 2018.

82. Feng, X. The Occurrence of Sarcopenia and Related Factors Analysis in Elderly Patients with Type 2 Diabetes Mellitus. Master's Thesis, Jiangsu Univeristy, Suzhou, China, 2016.

83. Ma, Y. The Occurence and Associated Factors of Sarcopenic Obesity in Older Adults. Master's Thesis, Tianjin University of Traditional Chinese Medicine, Tianjin, China, 2017.

84. Zhou, X.L.; Wang, S.; Xu, T.Y. Correlation between chronic atrophic gastritis and sarcopenia in elderly population. Med. J. Natl. Defending Forces Southwest China 2018, 28, 329-331. [CrossRef]

85. Zhang, T.; Gu, Y.H. Effect of abdominal obesity on sarcopenia and osteoporosis in elderly people with normal body mass index. Chin. J. Clin. Med. 2019, 26, 754-758. [CrossRef]

86. Yang, P.P. Investigation on the Current Status of Sarcopenia in Middle-Aged and Elderly Patients with T2DM. Master's Thesis, Nanchang University, Nanchang, China, 2019.

87. Beaudart, C.; Reginster, J.Y.; Slomian, J.; Buckinx, F.; Locquet, M.; Bruyère, O. Prevalence of sarcopenia: The impact of different diagnostic cut-off limits. J. Musculoskelet. Neuronal. Interact. 2014, 14, 425-431. [CrossRef]

88. Von Hippel, P.T. The heterogeneity statistic I(2) can be biased in small meta-analyses. BMC Med. Res. Methodol. 2015, 15, 35. [CrossRef]

89. Liu, W.; Chen, S.; Jiang, F.; Zhou, C.; Tang, S. Malnutrition and Physical Frailty among Nursing Home Residents: A Cross-Sectional Study in China. J. Nutr. Health Aging 2020, 24, 500-506. [CrossRef]

90. Pigłowska, M.; Guligowska, A.; Kostka, T. Nutritional Status Plays More Important Role in Determining Functional State in Older People Living in the Community than in Nursing Home Residents. Nutrients 2020, 12, 2042. [CrossRef]

91. Janssen, I.; Heymsfield, S.B.; Baumgartner, R.N.; Ross, R. Estimation of skeletal muscle mass by bioelectrical impedance analysis. J. Appl. Physiol. 2000, 89, 465-471. [CrossRef]

92. Yilmaz, O.; Bahat, G. Suggestions for assessment of muscle mass in primary care setting. Aging Male 2017, 20, 168-169. [CrossRef]

93. Chen, L.K.; Woo, J.; Assantachai, P.; Auyeung, T.W.; Chou, M.Y.; Iijima, K.; Jang, H.C.; Kang, L.; Kim, M.; Kim, S.; et al. Asian Working Group for Sarcopenia: 2019 Consensus Update on Sarcopenia Diagnosis and Treatment. J. Am. Med. Dir. Assoc. 2020, 21, 300-307.e2. [CrossRef]

94. Tyrovolas, S.; Koyanagi, A.; Olaya, B.; Ayuso-Mateos, J.L.; Miret, M.; Chatterji, S.; Tobiasz-Adamczyk, B.; Koskinen, S.; Leonardi, M.; Haro, J.M. Factors associated with skeletal muscle mass, sarcopenia, and sarcopenic obesity in older adults: A multi-continent study. J. Cachexia Sarcopenia Muscle 2016, 7, 312-321. [CrossRef] 